\title{
Inconspicuous on-Bed Respiratory Rate Monitoring
}

\author{
Ming-Chun Huang, Wenyao Xu, Jason Liu, 'Lauren Samy, \\ Amir Vajid, Nabil Alshurafa, and Majid Sarrafzadeh \\ Computer Science, University of California, Los Angeles \\ * Electrical Engineering, University of California, Los Angeles
}

\begin{abstract}
The monitoring of human respiratory rate is essential in many clinical applications including the detection and monitoring of sleep disorders, the monitoring of newborns for Sudden Infant Death Syndrome (SIDS), and identifying patients at high risk up to 24 hours before an adverse event like stroke and cardiac arrest [1]. Traditional noninvasive respiratory rate measurements in a hospital setting rely on clinical staff to visually track a patient's chest movement for a period of time to derive the respiratory rate from the number of movements observed. Failure to perform continuous and quantified measurements of respiratory rate could result in an inability to rescue a patient exhibiting respiratory distress. Severe after effects hinder recovery and result in loss of time, cost, or even life. This paper proposes an e-textile pressure sensitive bed sheet to non-invasively and accurately measure respiratory rate by analyzing time-stamped pressure distribution sequences. The bed sheet provides a 24/7 quantified on-bed respiratory rate monitoring service. It is made of e-textile and is similar to a regular bed sheet in comfort. As a result, it can seamlessly fit in common clinical or home environments, reducing the possible interference with a patient's regular sleeping habits and resulting in a type of inconspicuous monitoring.
\end{abstract}

\section{Keywords}

Respiratory Rate, Bedsheet, Pressure Sensor Array, Inconspicuous, Breath

\section{Categories and Subject Descriptors}

J.3 [Health and Medical information systems]: LIFE AND MEDICAL SCIENCE

\section{General Terms}

Design, Economics, Reliability, Experimentation

\section{INTRODUCTION}

Permission to make digital or hard copies of all or part of this work for personal or classroom use is granted without fee provided that copies are not made or distributed for profit or commercial advantage and that copies bear this notice and the full citation on the first page. Copyrights for components of this work owned by others than ACM must be honored. Abstracting with credit is permitted. To copy otherwise, or republish, to post on servers or to redistribute to lists, requires prior specific permission and/or a fee. Request permissions from Permissions@acm.org.

PETRA '13, May 29 -31 2013, Island of Rhodes, Greece

Copyright 2013 ACM 978-1-4503-1973-7/13/05 \$15.00

http://dx.doi.org/10.1145/2504335.2504353
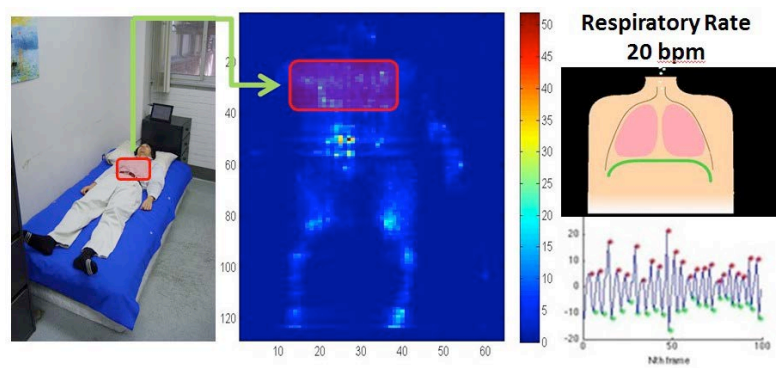

Figure 1: E-textile bedsheet (blue color) works as a transducer which converts pressure between human body to the bedsheet into voltage. Via analyzing the voltage matrix (pressure matrix), human body locations can be identified. When a subject sleeps on top of the bedsheet, its chest area should be detected. Based on analyzing the features (sum of pressure, standard deviation, and singular values), respiratory signals can be extracted. An example of the respiratory signal waveform is shown in the graph and the waveform can be visualized by a graphical user interface with bpm rate.

Respiratory rate is an important indicator of a person's overall health. It is important for many clinical uses and is often monitored during clinical evaluations as one of the four standard vital signs along with temperature, heart rate and blood pressure. Respiratory rate is not only important for detecting sleep disorders such as sleep apnea or SIDS, but it is also a significant predictor of deterioration and the onset of a cardiac arrest [2]. In fact, many studies have shown that an abnormally high respiratory rate (greater than 24 breath$\mathrm{s} / \mathrm{min}$ ) was observed in patients who experienced cardiac arrests or have been admitted to the ICU [3] [4]. Patients with even higher respiratory rates often died in hospital; the higher the respiratory rate, the higher the mortality rate. This is due to the fact that, in unstable patients, relative changes in respiratory rate are much greater than changes in heart rate or blood pressure, making respiratory rate a better and more accurate means of discriminating between stable patients and patients at risk [5].

In a typical hospital setting, respiratory rates are usually measured by manual observation by medical personnel or by attaching sensors to the patients' bodies to infer respiratory rate if continuous monitoring is required. In the former case, the measurement is often inaccurate due to the different ways of measuring respiratory rate. Measuring the respiratory rate of a patient using a stethoscope, for exam- 
ple, yields different results than manually tracking the movement of a patient's chest. Discrepancies were also reported between counting breaths for 30 seconds and then multiplying by 2 and counting breaths for 60 seconds [6]. Also, if the patients are aware that their respiratory rate is being monitored, they may involuntarily control their breathing, resulting in false measurements. In the latter case, extra sensor devices may need to be attached to the patients or placed on the bed, which might severely cause skin irritation and dramatically alter patients' regular sleeping habits.

In this paper, an e-textile bed sheet with a dense pressuresensitive sensor array system (Fig. 1) is introduced to measure human respiratory rate under any home/clinical environment. This system continuously detects a patient's pressure distribution on the bed. Respiratory rate and sleep positions could be extracted via analyzing time-stamped pressure map sequences. There was no special glue or attachment needed to be worn, allowing the test subjects to sleep freely and comfortably. The e-textile bed sheet was selected to be a natural human-sensor interface because it is close to a regular fabric-made bed sheet in feel and comfort. Test subjects were not even aware that they were under monitoring while they slept on top of this specially designed bed sheet. A comfortable and seamless monitoring system was desirable because sleep is a significant time for respiratory rate monitoring. According to the literature review, most emergencies associated with a high respiratory rate (cardiac arrest and heart attacks) are likely to occur during the last phase of sleep [7].

This design follows the concept of an inconspicuous monitoring system, which is a kind of sensing system that is embedded/integrated in existing commonly used daily life objects. This concept emphasizes the idea that no one's life or daily routine should be affected in any way as a result of using this sensing system. The worst scenario is where the new added functions are just not used; for example, bed sheet made of e-textile is still a fabric bed sheet if no power is provided. Therefore, a system that strictly follows this concept should be deployable to real life scenes seamlessly and immediately. To conclude, for a monitoring system to be inconspicuous, two requirements should be fulfilled: first of all, the improved sensing system should not be completely new to the user. Users should be familiar with the system from thorough everyday usage. Secondly, improved functions should be easily used and deployed to its targeted application without any extra setup or manual intervention.

The remainder of the paper is organized as follows: In section 2, previous research that was done to measure on-bed respiratory rate through various types of sensors is surveyed and discussed. Section 3 introduces both hardware and software architectures of the proposed system as well as the data acquisition flow. Section 4 describes respiratory rate extraction concepts and procedures. Experimental setup and results are presented in Section 5 and discussed in Section 6 . We conclude and describe our future work in Section 7.

\section{RELATED WORK}

Because measuring respiration rate accurately is a challenging task, there are not many sensor products commercially available to solve this problem. Most sensors are for use in hospitals and are part of a larger medical system that aggregates data from other sensors. One example is the Acoustic Respiration Rate monitor (RRa) designed by Masimo [8], which uses an adhesive sensor that is applied to the patient's neck while he or she is sleeping in order to measure his/her respiration rate. The sensor includes an integrated acoustic transducer and interprets the acoustic signal to identify inhalation and exhalation signals that represent a patient's breathing. Bates, et al. placed 3-axis MEMS sensors composed of accelerometers [9] and gyroscopes [10] on a patient's torso to continuously infer respiratory rate from the acceleration and angle acceleration information [11]. Although both authors consider their systems to be non-invasive, both systems interfere a patient's normal sleep because they require sensors to be attached to the body in order to operate.

Therefore, several research interested in noninvasive monitoring attempted to hide sensors from testing subjects. An example of such a system is the BAM Labs Touch-free Life Care (TLC) System [12]. This device consists of a mat that is placed under the bed mattress to sense the user's breathing rate. The device does not require any sensors to be attached to the user; therefore, the sleeping behavior of the test subjects should not be altered. However, the sensed data might contain a great deal of noise resulting from human body movements. The noise can also be affected by the shape/type of the mattress. Another similar past research placed a $40-\mathrm{kHz}$ ultrasound transmitter-and-receiver pair under a bed mattress for respiration rate monitoring. The transmitted ultrasound signal was reflected on the undersurface of the mattress and the amplitude of the received ultrasonic wave was modulated by the particular shape of the mattress [13]. These reflected signals were used to identify respiratory activity. This research should be considered an improvement because modeling the shape/type of the mattress made respiratory signals less relevant to the environmental conditions. However, human body movement was still an unavoidable source of noise which severely affected the respiratory rate accuracy.

Other past research focused on measuring respiration rate by placing sensors on certain body locations that exhibit significant variations caused by breathing movements. One example is to place a pressure sensor under a user's pillow. Xin et al. placed Water-filled vinyl tubes under a user's pillow to retrieve pressure signals from the user's breaths [14]. Signals were collected when the test subjects slept in supine and recumbent positions. The system used this unique pressure sensor $[15,16]$ to accurately measure the respiration rate of the users in these two well-controlled positions. The pillow must be placed under the head of the testing subjects and the human sleep postures are limited. To slightly relax the restriction of human movement, Townsend, et al. utilized an array of pressure sensors placed above the mattress to identify respiratory activity [17]. The device was a bed occupancy sensor with 24 pressure sensors. It was placed just below the pillow region to localize the areas of respiration. The authors had users sleep in prone, supine and side positions and asked them to simulate central apnea. The results showed that there was a correlation between pressure variance and occurrences of apnea. Using tiny pressure sensor arrays slightly relaxes the restriction of the human body movement, but a defined sleep posture was still required. 
This paper describes a system and a series of methods to utilize a full-size, e-textile-based high density pressure-sensitive bed sheet to solve all the issues mentioned above. 8192 pressure sensors were used to capture full body pressure distribution. With full body pressure information, human sleeping postures could be categorized and the corresponding chest location could be identified. Hence, there was no restriction on human sleeping habits. In addition, this e-textile-based bed sheet could be seamlessly integrated with existing mattresses without any changes. We refer to this type of sensor system design as an "inconspicuous monitoring system", which requires the system/algorithm design to be seamlessly integrated with commonly used objects and to have no interference or limitations on regular human behaviors.

\section{SYSTEM ARCHITECTURE}

E-textile-based hardware construction, respiratory signal extraction procedures, software operation, and the data acquisition process are described below.

\subsection{Hardware Architecture}

E-textiles are fabrics that have electronics and interconnections woven into them. They are perfectly suitable for home monitoring applications because of their flexibility, light weight and cost-effectiveness. E-textiles are just as comfortable and soft as regular fabrics but have one important difference that makes them suitable for serving as pressure sensors: the resistance of e-textiles changes when they are squeezed (by forces from their two sides).

\begin{tabular}{|l|l|}
\hline \multicolumn{2}{|c|}{ NORMAL RESPIRATORY RATES } \\
\hline Newborns & 44 respirations per minute \\
\hline Infants & $20-40$ respirations per minute \\
\hline Children (1-7 years) & $18-30$ respirations per minute \\
\hline Adults & $12-20$ respirations per minute \\
\hline
\end{tabular}

Figure 2: The normal respiratory rates table shows the respiratory rates among different age ranges. Four categories are listed: newborns, infants, children, and adults. As evident by the table, the human respiratory rate becomes fixed after the 7 th year of life. This is because the lung function and volume grow completely after that age. Newborns have the smallest lung volume and therefore also the highest respiratory rates in order to inhale enough oxygen for sustaining their metabolism.

The designed prototype bed sheet is a $2.5 \mathrm{~m} \times 1.25 \mathrm{~m}$ system containing $64 \times 128$ pressure sensors. 64 columns and 128 rows of conductive lines generate 8192 joint intersections. A piece of the e-textile fabric $[18,19]$ is located in between the row and column lines. Therefore, each joint intersection forms a pressure sensor made of a three-layer sandwiched structure. A sampling unit is connected to all conductive lines and performs matrix scanning to measure pressure map sequences. Retrieved pressure map signals from the 8192 sensors are quantified to an 8-bit integer matrix whose values range from 0 to 255, with 0 representing highest e-textile resistance/no pressure detected and 255 representing the lowest e-textile resistance/the maximum detectable pressure value $[20,21]$. The sampling rate is adjustable up to $10 \mathrm{~Hz}$, but $1.5 \mathrm{~Hz}$ was used for the respiratory rate measurement. This allows the system to achieve a maximum detectable breathing rate of up to 45 breaths per minute (bpm) according to the Nyquist rule. This upper bound on respiratory rate detection was more than sufficient for our application, since even newborns, with their small lung capacity and resulting frequent breathing, have a respiratory rate that does not exceed 44 breaths per minute as shown in Fig. 2.

\subsection{Software Architecture}

An Android application was created to acquire data from the bed sheet to a tablet. Analysis and visualization can be done locally if no network is available. The software flow is depicted by a state diagram shown in Fig 3. There are five sates related to the software operations: NO USER, INITIALIZATION, MOTION, RESPIRATION, and APNEA.

\begin{tabular}{|c|c|}
\hline STATE & DESCRIPTION \\
\hline NO USER & No user present \\
\hline INITIALIZATION & Waits for slope to settle and only then starts respiration detection algorithm \\
\hline RESPIRATION & Main state of the system that monitors and updates the user's RR \\
\hline APNEA & $\begin{array}{c}\text { User has not been breathing for a period of } 10 \text { seconds or longer or his/her } \\
\text { RR has fallen under } 9 \mathrm{bpm}\end{array}$ \\
\hline MOTION & User is moving \\
\hline
\end{tabular}

Figure 3: Software State Table

\subsubsection{NO USER: Initial System State}

The NO USER state is the initial state of the system which indicates that no user was sensed by the bed sheet. The system remains in the NO USER state as long as the sum of pressure values in the current frame is below an empirically determined threshold. From $\mathrm{P}=\mathrm{F} / \mathrm{A}$, where $\mathrm{F}$ is the weight of the subject and $\mathrm{A}$ is the surface area covered by his or her body, we could observe that pressure and subject weight are directly proportional. Therefore, the threshold for the NO USER state was derived from the pressure values of the subject with the lightest weight $(102 \mathrm{lbs})$ among our test subjects. To account for an even larger range of subjects, the threshold could be determined to be one third of the threshold of the lightest subject.

\subsubsection{INITIALIZATION: Identifying Users' Breaths}

Once the sum of the pressure values goes over the pre-set pressure threshold described above, the system state immediately changes to the INITIALIZATION state. As the label of the state indicates, this state is where the system initializes itself to get ready to detect subjects' breaths. During initialization, the system waits 10 seconds for the incoming signal to settle because the movements of getting on/off the bed are not relevant to the breath identification process. After the signal settles, the system starts the breath detection algorithm. After successfully detecting 3 breath$\mathrm{s}$ within a time interval of 20 seconds, corresponding to a minimum breathing rate of $9 \mathrm{bpm}$, the system enters the RESPIRATION state. This requirement ensures that only human beings, rather than heavy objects, could trigger the transition to the RESPIRATION state.

\subsubsection{RESPIRATION: Detecting Users' Breaths}


The RESPIRATION state is entered once a stable representation of the subject's breaths is identified. This state could be entered either from the INITIALIZATION state or from the MOTION state. An example of a respiratory signal is shown in Fig 1. A single oscillation represents a single breath made by the user. This is the case because a breath involves an inhalation period followed by an exhalation period which corresponds to increases and decreases in the pressure exerted on the bed sheet. Accurate identification of these oscillations was required to identify breaths. Once a single oscillation was determined to represent a single breath, the next step was to develop an algorithm to capture the occurrence of all breathing events and event intervals. This information can be used to calculate an instantaneous respiration rate and determine if the user was experiencing an apneic episode. The current respiratory rate is calculated as a moving average over the last 30 seconds (45 frames). A data structure was created to include occurrences of breaths over the last 45 frames and was used directly in the respiratory rate displayed to the end user. It was important to calculate the respiratory rate over a fixed amount of time so that any deviations in the respiratory rate could be accurately identified (e.g. apnea). In fact, when the respiratory rate falls below the chosen threshold $(9 \mathrm{bpm})$, the system changes its state from RESPIRATION to APNEA.

\subsubsection{APNEA: Respiratory Cessation}

There are two ways to enter the APNEA state: either the user's current respiration rate falls below $9 \mathrm{bpm}$ or no breaths are detected over a period of 10 seconds. This period was chosen because sleep apnea was clinically defined as the complete cessation of airflow for at least 10 seconds. To leave the APNEA state and transition back to the RESPIRATION state, the system has to detect at least 3 breaths over a period of 20 seconds. Again, this is an approximation of a breathing rate of $9 \mathrm{bpm}$.

\subsubsection{MOTION: Observing Users' Movements}

If a large amplitude of human movement occurs (due to a change in sleep posture during sleep), the state changes to MOTION. As in the INITIALIZATION state, the system waits until the pressure signal settles, but the waiting time is not pre-set in the MOTION state. Instead, a sleep posture detection algorithm, which is part of the breath detection algorithm, is executed continuously to identify when a user gets in a stable sleep posture. Once a stable sleep posture is recognized, the state changes back to RESPIRATION.

\subsection{Data Acquisition}

As mentioned in the previous section, this system is capable of recording pressure data acquired from the pressure sensitive bed sheet over time into a $\log$ file. When this record function is initiated, a comma-separated value (CSV) file with a time-stamped name is generated. Any new frame (64 X 128 integer pressure matrix) read from the bed sheet is stored to that $\log$ file. Each new row of a frame starts at the beginning of a new line, and the individual values of a single row are separated by commas. An additional blank line is printed between each data frame. The result is a CSV file that could be easily imported into MATLAB or R as a three dimensional matrix (frame number, row and column) for post-analysis.

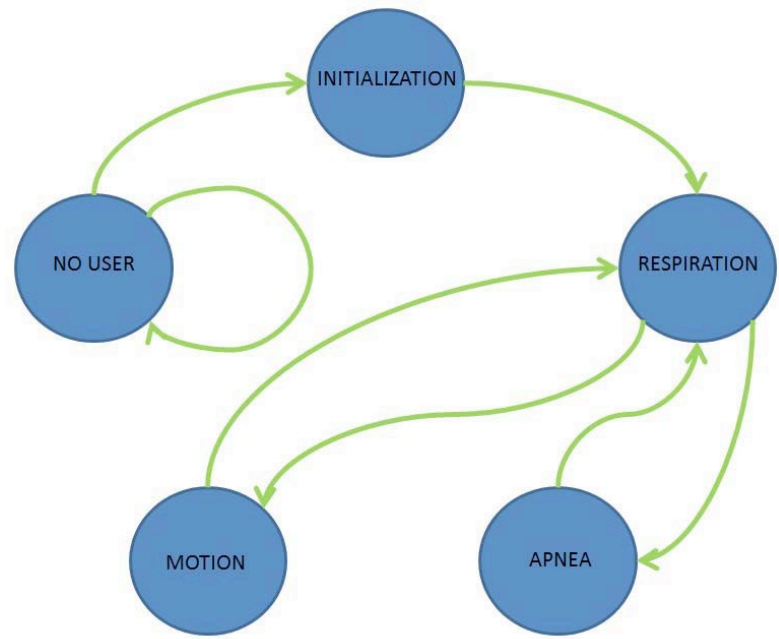

Figure 4: Software State Machine

Because the collected data was utilized to identify features that represented breaths of a typical subject, some type of ground truth was required to be synchronized with the current data acquisition framework. An extra button we added to the bed sheet system to provide such information. Every time the subject under test breathed, he/she would record the ground truth information by simply pressing the button. In general, the proposed bed sheet system did not need this button to measure respiratory rate. Overall, the system logs both sensor system events (bed sheet and ground truth) by saving the frame numbers and time of the annotated breaths to an additional $\log$.

\section{BREATH DETECTION ALGORITHM}

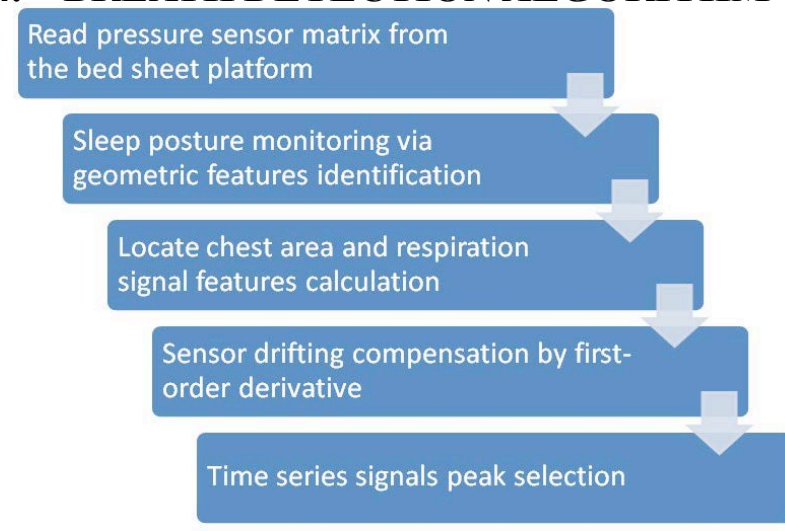

Figure 5: Algorithm flowchart for detecting respiration from the e-textile bedsheet

The breath detection algorithm is partitioned into five stages (Fig. 5). The first stage is to collect and format sensor data. Both bed sheet pressure data and the ground truth information are collected and aligned with the time-indexed frames. In order to extract better respiratory signal, the user's chest area needs to be localized. Geometric feature-based sleep posture recognition is applied to the raw pressure matrix sequences. Sleep posture recognition is a machine learning process described in the paper proposed by Jason et. al [20]. 
The main idea is to utilize the geometric information of the human body as features, such as the distance between chest and buttocks as well as human body symmetry information. In the respiration rate detection experiments, we directly exploit the sparse classifier parameters to identify sleep postures. If those sparse classifiers do not agree on a sequence of sleep postures derived from incoming pressure maps, the subject under test is identified to be in the MOTION state. In general, this sleep posture detection algorithm is robust to small movements and only gets confused by undefined sleep postures or body movements. This is because most of the body weight in a lying position is distributed in the torso area; thus, geometric features can easily capture static torso information even if the pressure among extremities varies or the subject body is relocated.
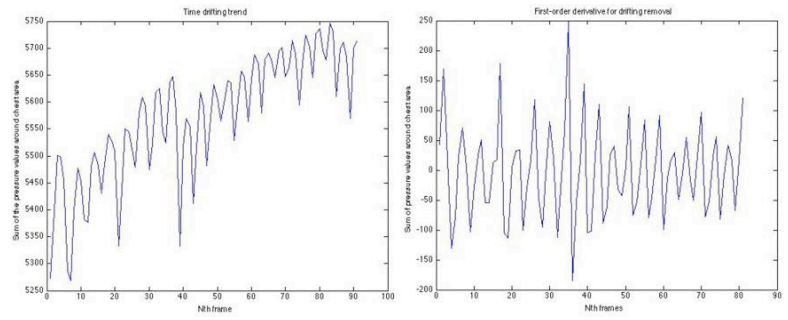

Figure 6: E-textile materials inherently suffer from the time drifting phenomenon. It is because e-textile material can be viewed as a textile-based leaky capacitor. Charges provided by the data acquisition controller should be stored and dissipated in the e-textile material. Therefore, even though there is no pressure value changes on the e-textile surface, the pressure readings collected from the controller may change over time. A simple first-order derivative method for digital signal processing can compensate the drifting and project the voltage signals into a horizonal plane.

Once the sleep posture is identified, chest location can be derived from the geometric features constraints. This geometricbased algorithm can continuously monitor and calculate the location of the chest for each incoming frame. Hence, the pressure values around the chest area can be extracted to increase the accuracy of breath detection and respiratory rate identification. Before extracting respiration-related features from the chest area, the time-drifting phenomenon of e-textile materials needs to be compensated [22]. Fig. 6 shows the drifting of the pressure sensor values of the chest area and the first-order derivative results. After drift compensation, four respiration-related features are analyzed in time series: the maximum pressure sensor value, the sum of all pressure sensor values, the standard deviation of all pressure sensor values and the maximum singular value of the chest area. The respiratory signal can be observed directly by searching for peek values of these time-series feature curves. To automate this process, a peek detection algorith$\mathrm{m}$ written in Matlab [23] is adopted to extract peek locations under some noise.

\section{EXPERIMENTS EVALUATION}

Experiments were designed to collect data from 14 different subjects. Mostly male subjects, ranging from 20 to 30 years of age, were recruited to participate in the study (Fig. 7). The participants were asked to sleep on top of the bed sheet on the ground, a soft mattress and a hard mattress in three different positions: prone (face down), supine (face up) and on their side. A total of at least 6 minutes of data was collected from each user ( 2 minutes per position) under 3 different mattress conditions. While the participants were sleeping, the system data acquisition framework was utilized to record their pressure data over time and create an annotated $\log$ of breathing events.

\begin{tabular}{|l|l|l|l|l|}
\hline Person\# & Gender & Height(inch) & Weight(lbs) & BMI \\
\hline 1 & Male & 67 & 173 & 27.09267 \\
\hline 2 & Male & 67 & 156 & 24.43039 \\
\hline 3 & Male & 75 & 165 & 20.62133 \\
\hline 4 & Male & 73 & 182 & 24.00938 \\
\hline 5 & Male & 66 & 176 & 28.40404 \\
\hline 6 & Female & 63 & 102 & 18.06652 \\
\hline 7 & Male & 65 & 160 & 26.62249 \\
\hline 8 & Female & 65 & 108 & 17.97018 \\
\hline 9 & Male & 70 & 140 & 20.08571 \\
\hline 10 & Male & 72 & 167 & 22.6468 \\
\hline 11 & Male & 69 & 143 & 21.1151 \\
\hline 12 & Male & 68 & 190 & 28.88625 \\
\hline 13 & Male & 68 & 150 & 22.80493 \\
\hline 14 & Male & 70 & 178 & 25.53755 \\
\hline
\end{tabular}

Figure 7: The anthropometric information of the 14 subjects (12 males and 2 females) participated in the data collection

\subsection{Respiratory-related Features Plotting with and without Chest Localization}

The first experiment attempted to test if chest localization helped in respiratory identification. 14 subjects were asked to statically lie on the bed in supine position in relax; thus, tiny body movements might randomly happen during the experiment. Nevertheless, there were no posture changes involved in this experiment. Compared to past research, the proposed bed sheet sensor system could capture full human body information through quantifying human body pressure distribution. Therefore, chest location information could be extracted with the geometric feature-based sleeping posture recognition algorithm. In order to prove whether chest localization helped in getting clean respiratory signals, two pressure value matrices were derived from the raw pressure value matrix sequence. One was a sub matrix which only related to each subject's chest area and the other one was the original pressure value matrix. Four respiration-related features were utilized to visualize waveforms from these two matrices. Fig. 8 shows the sampled curves of all four features and Fig. 9 shows a bar diagram that summarizes the respiratory signal identification results. The breath signal can be inferred from the chest area even via just manual identification (without any special analysis). This is because the movements around the chest area are related to the breath signal. Movements from other body parts simply disturb the respiration-related features. 
Full Bed Sheet Area
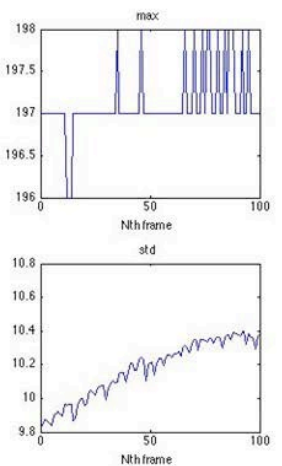
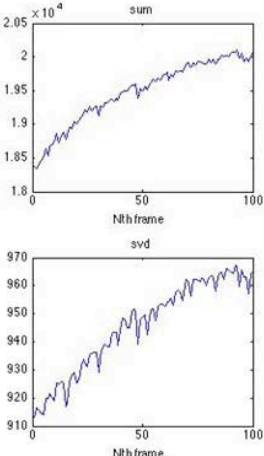

Chest Only Area
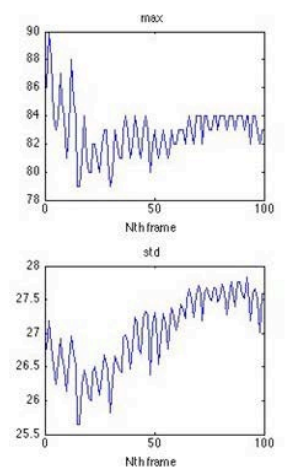

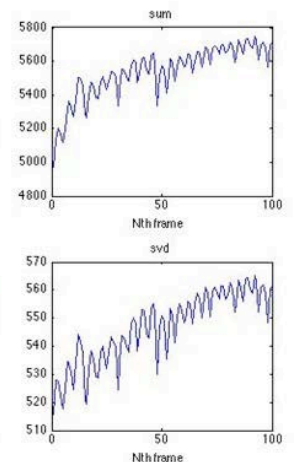

Figure 8: This experiment emphasizes the importance of using an array-based bedsheet rather than sparse sensors on the bed. The respiratory signals become more prominent in the chest area than the full body. The chest area can be identified with high density array-based bedsheets. This information can assist respiratory signal detection. Four features (max of pressure, sum of pressure, standard deviation of the pressure and singular value of the pressure) are calculated on both the full bed sheet area and the chest area. The singular value feature tends to provide the most reliable information for breath rate calculation.

\subsection{Respiratory-related Features versus Sleep- ing Postures}

Human sleep postures are usually not fixed during sleeping. This experiment attempted to demonstrate that the proposed bed sheet system could identify a respiratory signal in the supine, prone, and side positions. In addition, the test subject could sleep on any corner of the bed sheet without any restriction. Human pressure distribution maps can be aligned by the center of weight and eigenvectors of the sleep orientation. Although the respiratory signals were always captured, different postures indeed generated different pressure distributions on the bed sheet when the subject breathed. Respiration-related features could still be visually compared with the ground truth in the prone and supine postures. However, since, in the side postures, the contact area between the human body and the bed sheet is the ar$\mathrm{m}$ area or the side of the body rather than the chest, side postures tended to obscure the breath signal. Therefore, even if breathing made the chest area expand and shrink, the pressure on the bed sheet only changed slightly. Fig. 9 summarizes the experiments and provides accuracy analysis among these sleep postures.

\subsection{E-textile Sensor Drifting and the Corre- sponding Compensation}

In the algorithm section, we described the drifting phenomenon of the respiration-related features. This phenomenon was not caused by a measurement error, but rather by the nature of the e-textile-based sensor. E-textiles tend to continuously decrease their resistance if there is a load on top. Therefore, to compensate for resistance drifting, first order derivation was exploited to remove the slope of the respiration-related feature curves. Fig. 10 shows accuracy improvements after first order derivation compensation.

\subsection{Breath Event Identification with Peak De- tection Algorithm}

Although the respiration-related features seemed to be visually well-aligned with the ground truth, automated res-

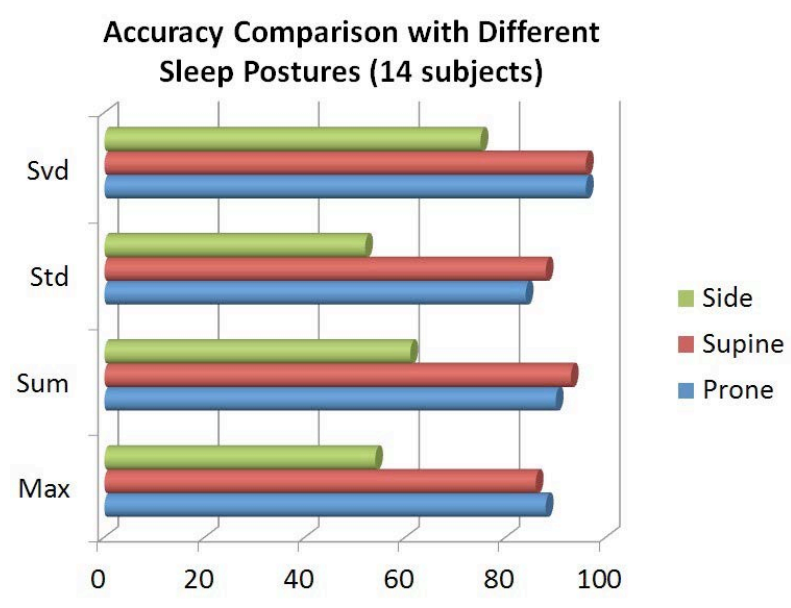

Figure 9: In order to provide continuous respiratory rate monitoring, the effect of different sleep postures (side, supine, and prone) on the proposed system is analyzed. Supine and prone postures, on average, are the most appropriate postures for measuring respiratory rate, because the chest location of these two postures is easier to identify. We can only estimate the chest location for the side posture through the location of the arm, but it is usually harder to be seen in the collected pressure images.

piratory rate monitoring was still required for continuous on-bed monitoring. A peak detection algorithm written in MATLAB was used to identify the peak locations in the feature curves and then infer the respiratory activity. The results are shown in Fig. 9.

\section{DISCUSSION AND FUTURE WORK}

The series of experiments mentioned above provides a clear demonstration of the functionality of the current e-textile bed sheet design. Chest localization helps to extract a cleaner respiratory signal and the full-size bed sheet captures full human pressure distribution information; therefore, exper- 


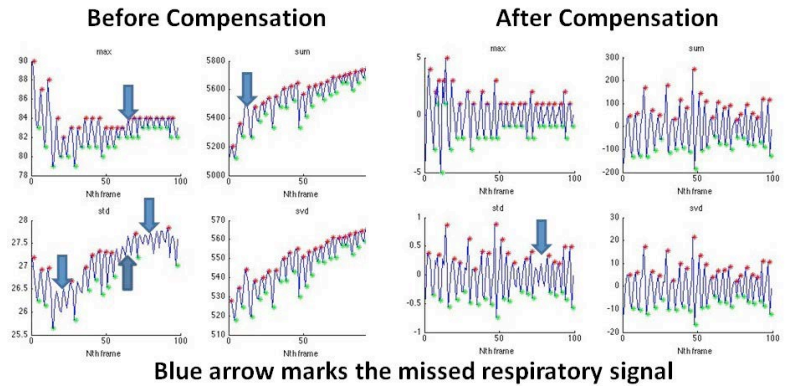

Figure 10: This experiment demonstrates that drifting compensation can effectively facilitate respiratory rate monitoring. Blue arrows mark the missed respiratory signals. After compensation, the number of missed detection drops significantly. We observe that the waveform quality from the single value feature is remarkable. Even without compensation, there is no missed detection for the single value feature.

\section{Average Accuracy Compared with Ground Truth (14 subjects)}

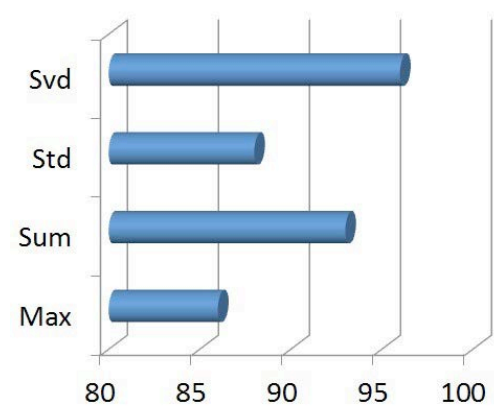

- Average Accuracy Compared with Ground Truth (14 subjects)

Figure 11: This figure shows that the singular value feature is best feature in the experiments when comparing the identified respiratory signals with the manually collected breathing ground truth. On the other hand, $\max$ pressure value is the most unreliable feature in the experiments.

iments or clinical trials can be performed with very little manual intervention. In addition, human sleep posture can be directly inferred from the pressure distribution. Hence, when a test subject changes his/her sleep posture, the corresponding frame number should be marked. Breath signals extracted at that stage should be marked as invalid because changing postures generates a huge amount of human pressure variation and this variation is not relevant to the respiratory rate. From the experiments, we also realize that side postures tend to cause low-quality respiratory signals. Failure to identify sleep postures and chest locations may misinterpret the respiratory signals collected from continuously automatic monitoring. After all, no one can keep the same sleep posture or position throughout a whole night. The proposed design effectively solves these issues and provides desirable measuring results. Nevertheless, in the following subsections, we want to further investigate how much accuracy can be obtained while the subject changes his/her sleep posture. Also, we want to investigate whether or not the type of mattress underneath causes different respiratory rate results.

\subsection{Effect of Human Movement}

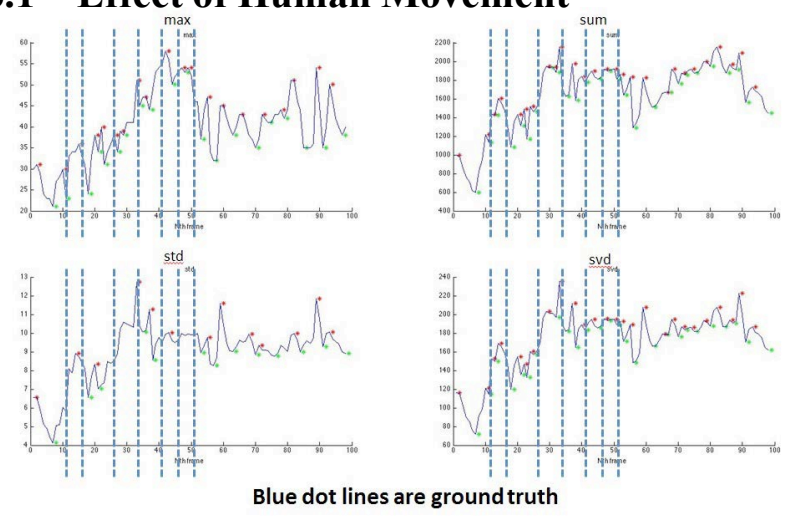

Figure 12: The respiratory rate monitoring system tends to fail when the subject under test is moving. The raw pressure feature data and the corresponding ground truth information are provided. Fortunately, apnea and other highly dangerous respiratory diseases commonly happen when the patient is not moving. Therefore, we can safely leave this problem for future work and still use this system to provide a respiratory rate monitoring service.

In this experiments, test subjects are asked to begin with a supine sleep posture, then slightly move their body, and then change their position to side or prone. In order to extract the best possible respiratory information, standard deviation of the chest area is exploited as a respiration-related feature. Fig. 12 summarizes the experimental results. From the results, we can realize that measuring respiratory rate when the subject is changing his/her sleep position is not practical. Nevertheless, measuring respiratory rate of moving subject may not be necessary. The proposed design which can track sleep posture and position is sufficient for continuous on-bed respiratory rate monitoring.

\subsection{Effect of Measurement Environment} Accuracy Analysis under Different Mattress Environment

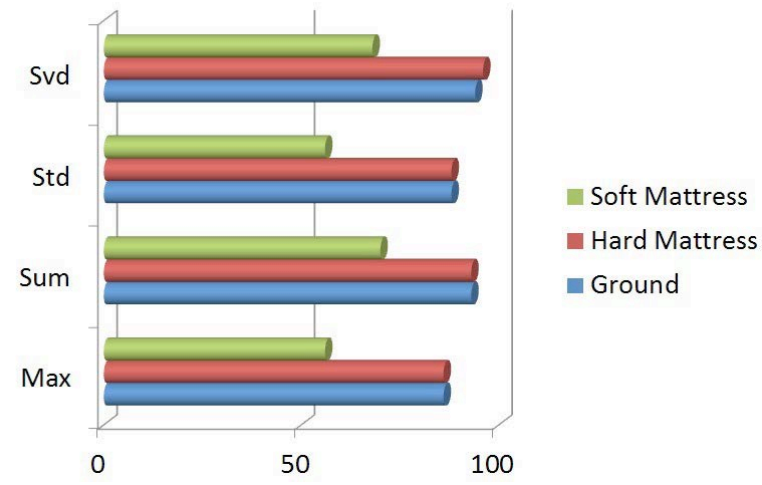

Figure 13: The stiffness of the mattress under the bed sheet may affect respiratory rate monitoring as well. This bed sheet works well on the ground and hard mattress. In order to deploy this system in clinics or hospitals, better hardware design or signal extraction techniques need to be explored in order to generate good results from soft mattresses.

In order to deploy the bed sheet to the home/clinical environment, we should test if different mattresses change the 
respiratory monitoring results. The bed sheet was deployed to different environments such as ground, hard mattress (default in all the experiments), and soft mattress. Fig. 13 shows that harder surfaces seem to be better because soft surface can absorb some of the pressure from the human breathing.

\subsection{Procedures to Handle Respiratory Detec- tion Failure}

From the discussion above, the proposed system can still make inaccurate measurements if the subject under test is moving or is in a side position. Also the mattress type does indeed cause some differences in measuring. Therefore, the respiratory prediction for a moving or side-positioned subject should be marked and manually examined. In addition, prior knowledge about normal breathing, from 3 times in 20 seconds to 24 times in 60 seconds, should be taken into consideration. This prior knowledge highly depends on the subject's age [24].

\section{CONCLUSION AND FUTURE WORK This}

paper has presented a new inconspicuous monitoring design for accurately measuring on-bed respiratory rate. High density pressure sensor maps are built from e-textile material. An e-textile-based bed sheet is similar to a regular cotton-made bed sheet. Therefore, it can be seamlessly integrated in home/clinical environments and reduces possible interferences to patients' regular sleeping habits. In addition, this bed sheet sensor can capture full human body pressure distribution, locate a subject's chest, and map pressure variations to respiratory rate signals.

\section{ACKNOWLEDGEMENT}

This work is partially supported by NSF grant IIS- 1329119.

\section{REFERENCES}

[1] M. Cretikos and J. Chen and K. Hillman and R. Bellomo and S. Finfer and A. Flabouris, "The objective medical emergency team activation criteria: a case-control study," Resuscitation, vol. 73, no. 1, pp. 62-72, 2007.

[2] TJ. Hodgetts and G. Kenward and IG. Vlachonikolis and S. Payne and N. Castle, "The identification of risk factors for cardiac arrest and formulation of activation criteria to alert a medical emergency team," Resuscitation, vol. 54, no. 2, pp. 125-131, 2002.

[3] JF. Fieselmann and MS. Hendryx and CM. Helms and DS. Wakefield, "Respiratory rate predicts cardiopulmonary arrest for internal medicine patients," J Gen Intern Med, vol. 8, no. 7, pp. 354-360, 1993.

[4] DR. Goldhill and AF. McNarry and G. Mandersloot and A. McGinley, "A physiologically-based early warning score for ward patients: the association between score and outcome," Anaesthesia, vol. 60, no. 6, pp. 547-553, 2005.

[5] CP. Subbe and RG Davies and E Williams and P. Rutherford and L. Gemmell, "Effect of introducing the modified early warning score on clinical outcomes, cardio-pulmonary arrests and intensive care utilisation in acute medical admissions," Anaesthesia, vol. 58, no. 8, pp. 797-802, 2003.
[6] S. Fleming, "Measurement and fusion of non-invasive vital signs for routine triage of acute paediatric illness," Master's thesis, University of Oxford, 2010.

[7] L. Blue, "When Are You Most Likely to Have a Heart Attack." http://www.time.com/time/health/article/0, $8599,1825044,00 . \mathrm{html}$.

[8] "Acoustic Respiration Rate(RRa) Masimo Corporation." http: //www.masimo.com/rra/index.htm.

[9] M.-C. Huang, E. Chen, W. Xu, M. Sarrafzadeh, B. Lange, and C.-Y. Chang, "Gaming for Upper Extremities Rehabilitation," in ACM/EMS Conference on Wireless Health, Oct.

[10] M.-C. Huang, W. Xu, Y. Su, C.-Y. Chang, B. Lange, and M. Sarrafzadeh, "Smart Glove for Upper Extremities Rehabilitative Gaming Assessment," in International Conference on Pervasive Technologies Related to Assistive Environments, Jun. 2012.

[11] A. Bates, "Respiratory Rate and Flow Waveform Estimation from Tri-axial Accelerometer Data," in Body Sensor Networks (BSN), 2010 International conference on, pp. 144-150, IEEE press, 2010.

[12] "BAM Labs Touch-free Life Care (TLC) System. BAM Labs, Inc.." http://www.bamlabs.com/product/.

[13] Y. Yamana and S. Tsukamoto and K. Mukai and H. Maki and $\mathrm{H}$. Ogawa and Y. Yonezawa, "A sensor for monitoring pulse rate, respiration rhythm, and body movement in bed," in Conf Proc IEEE Eng Med Biol Soc, pp. 5323-5326, IEEE Press, 2011.

[14] X. Zhu and W. Chen and T. Nemoto and Y. Kanemitsu and K. Kitamura and K. Yamakoshi and D. Wei, "Real-time monitoring of respiration rhythm and pulse rate during sleep," IEEE Trans Biomed Eng, vol. 53, no. 1, pp. 2553-2563, 2006.

[15] W. Xu, M.-C. Huang, N. Amini, J. Liu, L. He, and M. Sarrafzadeh, "Smart Insole: A Wearable System for Gait Analysis," in International Conference on Pervasive Technologies Related to Assistive Environments, Jun. 2012

[16] M.-C. Huang, W. Xu, J. Liu, L. He, Y. Su, and M. Sarrafzadeh, "Inconspicuous Personal Computer Protection with Touch-Mouse," in International Conference on Human Computer Interaction, 2013.

[17] DI. Townsend and M. Holtzman and R. Goubran and M. Frize and F. Knoefel, "Simulated central apnea detection using the pressure variance," in Conf Proc IEEE Eng Med Biol Soc, pp. 3917-3920, IEEE press, 2009.

[18] W. Xu and Z. Li and MC. Huang and N. Amini and M. Sarrafzadeh, "ecushion: an etextil device for sitting posture monitoring," in Body Sensor Networks (BSN), 2011 International conference on, pp. 194-199, IEEE press, 2011.

[19] Wenyao Xu and Ming-Chun Huang and Navid Amini and Lei He and Majid Sarrafzadeh, "Smart cushion: Design and calibration of textile sensors for sitting posture analysis," IEEE Sensors Journal, 2013.

[20] J. Liu and W. Xu and MC. Huang and N. Alshurafa and M. Sarrafzadeh, "A Dense Pressure Sensitive Bedsheet Design for Unobtrusive Sleep Posture Monitoring," in Pervasive Computing and Communications (PerCom), 2012 IEEE International Conference on, IEEE press, 2013.

[21] J. Liu, M.-C. Huang, W. Xu, N. Alshursfa, and M. Sarrafzadeh, "On-bed Monitoring for Range of Motion Exercises with a Pressure Sensitive Bedsheet," in IEEE Conference on Body Sensor Network, 2013.

[22] J. Meyer, "Textile Pressure Sensor: Design, Error Modeling and Evaluation," Master's thesis, ETH Zurich, 2008.

[23] "peakdet: Peak detection using MATLAB." http://www.billauer.co.il/peakdet.html.

[24] "Delmar's Comprehensive Medical Assisting: Administrative and Clinical Competencies." http: //books.google.com/books? id=AUhJKmKJ_eEC\&pg= $P A 573 \# v=o n e p a g e \& q \& f=f a l s e$. 